\title{
An Enhanced Spring-Particle Model for Red Blood Cell Structural Mechanics: Application to the Stomatocyte-Discocyte- Echinocyte Transformation
}

\author{
Mingzhu Chen \\ Technological University Dublin, 452413@tudublin.ie \\ Fergal Boyle \\ Technological University Dublin, fergal.boyle@tudublin.ie
}

Follow this and additional works at: https://arrow.tudublin.ie/engschmecart

Part of the Biomaterials Commons, and the Biomechanics and Biotransport Commons

\section{Recommended Citation}

Chen, M. \& Boyle, F. (2017)An Enhanced Spring-Particle Model for Red Blood Cell Structural Mechanics: Application to the Stomatocyte-Discocyte- Echinocyte Transformation, Journal of Biomechanical Engineering, 19-August-17. DOI: 10.1115/1.4037590

This Article is brought to you for free and open access by the School of Mechanical and Design Engineering at ARROW@TU Dublin. It has been accepted for inclusion in Articles by an authorized administrator of ARROW@TU

Dublin. For more information, please contact

arrow.admin@tudublin.ie, aisling.coyne@tudublin.ie, gerard.connolly@tudublin.ie.

Funder: Technological University Dublin

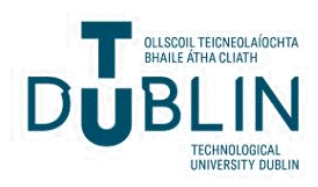


AQ1

AQ2

\section{An Enhanced Spring-Particle Model for Red Blood Cell Structural Mechanics: Application to the Stomatocyte-Discocyte- Echinocyte Transformation}

Red blood cells (RBCs) are the most abundant cellular element suspended in blood. Together with the usual biconcave-shaped RBCs, i.e., discocytes, unusual-shaped RBCs are also observed under physiological and experimental conditions, e.g., stomatocytes and echinocytes. Stomatocytes and echinocytes are formed from discocytes and in addition can revert back to being discocytes; this shape change is known as the stomatocyte-discocyte-echinocyte (SDE) transformation. To-date, limited research has been conducted on the numerical prediction of the full SDE transformation. Springparticle $R B C(S P-R B C)$ models are commonly used to numerically predict $R B C$ mechan$i$ ics and rheology. However, these models are incapable of predicting the full SDE transformation because the typically employed bending model always leads to numerical instability with severely deformed shapes. In this work, an enhanced SP-RBC model is proposed in order to extend the capability of this model type and so that the full SDE transformation can be reproduced. This is achieved through the leveraging of an advanced bending model. Transformed vesicle and RBC shapes are predicted for a range of reduced volume and reduced membrane area difference (MAD), and very good agreement is obtained in the comparison of predicted shapes with experimental observations. Through these predictions, vesicle and SDE transformation phase diagrams are developed and, importantly, in the SDE case, shape boundaries are proposed for the first time relating $R B C$ shape categories to $R B C$ reduced volume and reduced $M A D$.

[DOI: $10.1115 / 1.4037590$ ]

\section{Introduction}

Red blood cells (RBCs) are the most abundant cellular element suspended in blood. A normal RBC count in a healthy individual is about $5,000,000 / \mathrm{mm}^{3}$, accounting for $99 \%$ of all suspended cellular elements or $45 \%$ of the total blood volume. These RBCs are highly deformable and, during their lifespan of about 120 days, repeatedly travel through blood vessels with diameters as small as $3 \mu \mathrm{m}$ delivering oxygen in the circulatory system [1]. A healthy RBC has a biconcave shape with a diameter of $8 \mu \mathrm{m}$, a thickness of $2 \mu \mathrm{m}$, and a volume of $65 \%$ of a sphere with the same surface area. Structurally, a RBC is a liquid-core membrane-bounded capsule. The liquid, known as the cytoplasm, is a mixture of hemoglobin and enzymes and is generally considered as a Newtonian fluid [2]. The membrane is a composite material consisting of an outer layer of plasma membrane (PM) and an inner cytoskeleton layer. The PM consists mainly of a lipid bilayer which offers no resistance to in-plane shearing, a small resistance to out-of-plane bending but a high resistance to surface area dilation. The cytoskeleton is a nonlinear hyper-elastic-behaving network, attached to the PM via anchoring proteins, conferring structural integrity to the RBC membrane (see Fig. 1).

Besides the normal biconcave-shaped RBCs, known as discocytes, some unusual-shaped RBCs are also observed under physiological [3-5] and experimental conditions [6-8], e.g.,

${ }^{1}$ Corresponding author

Manuscript received May 30, 2017; final manuscript received July 13, 2017; published online xx xx, xxxx. Assoc. Editor: Guy M. Genin. stomatocytes and echinocytes. Instead of having a biconcave shape, stomatocytes have a single invagination, while echinocytes have spicules evenly distributed on the membrane surface. Stomatocytes and echinocytes are formed from discocytes and can revert back to being discocytes; this shape change is known as stomatocyte-discocyte-echinocyte (SDE) transformation. The SDE transformation can be induced by a number of factors, e.g., deviations from healthy ranges of $p \mathrm{H}$ and cholesterol levels. A deviation from the healthy range of $p \mathrm{H}$ level promotes ionic interactions on the PM surface $[9,10]$, while a deviation in the cholesterol level promotes lipid and protein exchanges between the two leaflets of the PM bilayer [11]. SDE transformation inducing factors lead to a change in the volumes of the bilayer leaflets, and since the leaflet thicknesses are constant, these volume changes result in a change of the area difference between the outside surface of the outer layer and inside surface of inner layer, i.e., a change of the membrane area difference (MAD). In other words, every particular RBC shape in the SDE transformation is associated with a unique combination of MAD, volume and area, and with the large variety of SDE transformation shapes possible, the use of the MAD, in addition to the volume and area, allows unique RBC shapes to be identified.

To-date, limited research has been conducted on the numerical simulation of the full SDE transformation. Lim et al. [12] simulated the full transformation of a normal RBC. This simulation study assessed the effects of both the MAD and the equilibrium cytoskeleton shape on the SDE transformation for one volume only, i.e., the normal RBC volume. In this study, the RBC membrane was modeled using the Skalak (SK) constitutive law [13] 50 51 51 52 53 53
54
55 55 56
57 57 58 59 (1) 65 65 66
67 


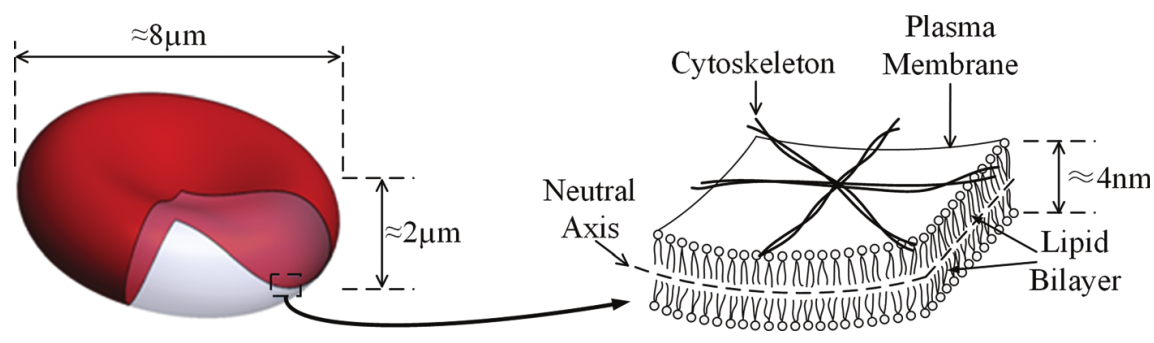

Fig. 1 Schematic diagrams of (a) a healthy RBC and (b) the RBC membrane. The PM consists mainly of a lipid bilayer, while the cytoskeleton is a hyper-elastic-behaving network attached to the PM via anchoring proteins.

and the modeled surface was discretized using a mesh of regular triangular elements. The transformed shapes were predicted using the Monte Carlo method to ensure that stable equilibrium shapes were obtained. Through simulations, it was concluded that the SDE transformation can be induced by varying the MAD and, importantly, it was proposed that the equilibrium cytoskeleton shape is likely to be an ellipsoid rather than the widely adopted biconcave shape. A similar simulation study was completed by Khairy and Howard [14] where, in addition to the SDE transformation, vesicle transformation was also simulated. One of the aims of this study was to demonstrate the computational efficiency of the spherical harmonics parameterization method which was employed to predict the transformed shapes. In the study, both the vesicle and RBC membrane were modeled using continuum mechanics, i.e., the SK constitutive law [13]. Based on the results obtained, the authors claimed that their method was highly computational efficient compared with other modeling approaches.

In both the simulation studies by Lim et al. [12] and Khairy and

Howard [14], the RBC membrane was modeled using a continuum constitutive law, i.e., a continuum model, assuming the membrane was a homogeneous isotropic thin shell. In addition, an areadifference-elasticity (ADE) contribution was introduced into their membrane models to include the effect of the MAD change on the SDE transformation. As an alternative to using continuum models, some researchers have employed spring-network-based models to numerically simulate the RBC membrane [15-18]. These networks consist of a number of nonlinear springs arranged in a triangle-based topology. These RBC models are known as springparticle (SP) RBC models and are widely used to study RBC mechanics [15], rheology [16], and RBC-related diseases [19]. SP-RBC models are mathematically simpler than continuum models and have extensive modeling capabilities. Diseased RBC dynamics [20] and RBC fluctuations [21,22] have been successfully simulated using SP-RBC models, while no similar simulations have been conducted using continuum models. However, the Spring-particle RBC (SP-RBC) models developed to-date are incapable of simulating the SDE transformation while the continuum-based models are. This deficiency is due to the bending model typically employed, which is based on the subtended angle between neighboring network triangular elements. Use of this bending model to predict the full SDE transformation leads to numerical instability always when modeling echinocytes and sometimes with stomatocytes. The use of very fine meshes does not alleviate the instability, even when using a very fine mesh with 60,000 spring elements. Mesh reconstruction may be a solution but would render the model very demanding computationally.

Inspired by previous research $[12,14]$, an enhanced SP-RBC model is proposed here in order to improve the capability of the conventional ones. This SP-RBC model is more advanced than the conventional ones $[23,24]$ from two perspectives. First, the bending model, which uses surface curvature, is independent of the network topology. Second, an ADE contribution, based on the MAD change, is included. This enhanced SP-RBC model is used to predict the transformed shapes of vesicles and RBCs resulting from changes to the model volume and MAD. Predictions of transformed vesicle shapes facilitate an examination of the accu- 123 racy and stability of the bending model, since vesicle transforma- 124 tion is driven mainly by minimization of the bending energy. 125 Importantly, the RBC predictions can be considered as a follow- 126 on to the work of Lim et al. [25], with an extensive investigation 127 of the effect of changing the RBC volume completed. In addition, 128 a direct comparison of vesicle and RBC transformed shapes yields 129 RBC shape boundaries, which relate RBC shape categories to 130 $\mathrm{RBC}$ reduced volume and reduced MAD. In Sec. 2, the structural 131 mechanics employed by the SP-RBC model are introduced along 132 with the methodology employed for predicting a RBC equilibrium 133 shape. In Sec. 3, results of two test cases are presented. The first 134 test case involves the prediction of transformed vesicle shapes, 135 while the second test case involves the prediction of the full SDE 136 transformation resulting from changes to the RBC volume and 137 MAD.

\section{Spring-Particle Red Blood Cell Model}

The SP-RBC model employs an area and volume conserving 140 spring network to predict a RBC equilibrium structural geometry. 141 As the model has been developed to predict steady-state geome- 142 tries only, the viscous behavior of the PM does not need to be 143 accounted for.

Geometry of the RBC Model. A healthy RBC is biconcave in $\mathbf{1 4 5}$ shape. The average RBC surface geometry can be approximated 146 by the equation [26]

$z= \pm D_{o}\left(1-\frac{4\left(x^{2}+y^{2}\right)}{D_{o}^{2}}\right)^{1 / 2}\left(a_{0}+a_{1} \frac{x^{2}+y^{2}}{D_{o}^{2}}+a_{2} \frac{\left(x^{2}+y^{2}\right)^{2}}{D_{o}^{4}}\right)$

where $x, y$, and $z$ are the Cartesian coordinates; $D_{o}$ is the initial 148 diameter of the RBC with a value of $7.82 \mu \mathrm{m}$; and $a_{0}, a_{1}$, and $a_{2} 150$ are constants with values of $0.0518,2.026$, and -4.491 , respec- 151 tively. This equation is employed in SolidWorks (SolidWorks 152 Corp., Concord, MA) to generate a 3D solid model. The surface 153 of this solid model is subsequently discretized using Ansys (Ansys 154 Inc., Canonsburg, PA) to obtain a mesh of triangular elements. 155 This mesh network forms the spring-particle system, where the 156 element boundaries are considered the springs and the element 157 nodes considered the particles. This spring-particle system repre- 158 sents the neutral plane of the RBC membrane, located at the inter- 159 face of the inner and outer leaflet surfaces of the PM bilayer, see 160 Fig. 1.

Helmholtz Free Energy of the RBC Model. Structurally, a 162 $\mathrm{RBC}$ is a liquid-core membrane-bounded capsule. The liquid, 163 known as the cytoplasm, is an incompressible Newtonian fluid 164 with constant volume. The membrane has a dual-layer structure 165 consisting of the PM and the cytoskeleton. Mechanically, the PM 166 confers resistance to surface area dilation and out-of-plane 167 
168 bending, while the cytoskeleton confers resistance to in-plane 169 shearing. Correspondingly, the Helmholtz free energy of the RBC 170 model has four constituent elements: energies due to constraints 171 of the model volume and membrane surface area, out-of-plane 172 bending, and in-plane shearing, i.e.,

$$
E_{\mathrm{Total}}=E_{\mathrm{VC}}+E_{\mathrm{AC}}+E_{B}+E_{\mathrm{S}}
$$
of the RBC model is given by

$$
E_{\mathrm{VC}}=\frac{K_{V}}{2 V_{o}}\left(V-V_{o}\right)^{2}
$$

where $K_{V}$ is the volume-constraint modulus [27,28] and in this work is set as $1000 \mathrm{~N} / \mathrm{m}^{2} . V$ and $V_{o}$ are the RBC-model instantaneous and reference volumes, respectively.

The PM surface-area constraint energy consists of two terms. The first one is due to the movement restriction imposed by the anchoring proteins that attach the cytoskeleton to the PM, while the second one is due to the surface-area incompressibility of the PM, i.e.,

$$
E_{\mathrm{AC}}=\frac{K_{\mathrm{LA}}}{2} \sum_{k=1}^{N_{T}} \frac{\left(A_{k}-A_{k, o}\right)^{2}}{A_{k, o}}+\frac{K_{\mathrm{GA}}}{2 A_{o}}\left(A-A_{o}\right)^{2}
$$

where $K_{\mathrm{LA}}$ and $K_{\mathrm{GA}}$ are the local and global area dilation moduli $[27,28]$ which are set as $10^{-4} \mathrm{~N} / \mathrm{m}$ and $10^{-3} \mathrm{~N} / \mathrm{m}$, respectively, $k$ is triangular element index, $N_{\mathrm{T}}$ is the total number of triangles, $A_{k}$ and $A_{k, o}$ are instantaneous and the reference areas of triangle $k$, respectively, and $A$ and $A_{o}$ are instantaneous and reference surface areas of the RBC model, respectively.

Bending Energy. Typically, the bending model employed by conventional SP-RBC models is an approximation to the Helfrich bending model [25] resulting from a number of assumptions, see the Appendix. Importantly, the bending model is based on the subtended angle between neighboring triangular elements and is mesh dependent [29]. Consequently, the conventional SP-RBC model is incapable of simulating the SDE transformation, because the bending model causes numerical instability as the model membrane undergoes severe deformation.

The membrane bending model employed in this work is based on the one previously employed by Lim et al. [12]. This bending model energy consists of two terms. The first term is due to the local bending resistance, while the second term is due to the membrane ADE contribution [25]. In contrast to the conventional one, the employed bending model is based on surface curvature, i.e.,

$$
E_{B}=\frac{K_{\mathrm{LB}}}{2} \sum_{i=1}^{N_{P}}\left[A_{i}\left(C_{i}-C_{i, o}\right)^{2}\right]+\frac{K_{\mathrm{GB}}}{H^{2} A_{o}}\left[\Delta A-\Delta A_{o}\right]^{2}
$$

where $K_{\mathrm{LB}}$ and $K_{\mathrm{GB}}$ are the RBC-membrane local and global bending moduli [25], respectively, with the same default value of $2.5 \times 10^{-19} \mathrm{~N} \cdot \mathrm{m}, \mathrm{i}$ is particle index, $N_{P}$ is the total number of particles, $A_{\mathrm{i}}$ is area occupied by particle $i, C_{\mathrm{i}}$ and $C_{\mathrm{i}, o}$ are instantaneous and reference curvatures of particle $i$, respectively, $H$ is the membrane thickness with a value of $4 \times 10^{-9} \mathrm{~m}$, and $\Delta A$ and $\Delta A_{o}$ are the instantaneous and reference MADs, respectively. The particle area, $A_{\mathrm{i}}$, is one-third of the total area of the triangles that share particle $i$ and the curvature, $C_{\mathrm{i}}$, is calculated as

$$
C_{i}=\frac{\frac{1}{2} \sum_{j=1}^{N_{w}}\left(\theta_{j} L_{j}\right)}{A_{i}}
$$

where $j$ is the element edge index, $N_{W}$ is the total number of edges 223 which share particle $i, L_{j}$ is the length of edge $j$, and $\theta_{j}$ is the angle 224 between the triangles which share edge $j$, see Fig. 2 .

The MAD, $\Delta A$, is related to the curvature as follows:

$$
\Delta A=H \sum_{i=1}^{N_{p}}\left(C_{i} A_{i}\right)
$$

By substituting Eq. (7) into Eq. (5), it is interesting to see that the 228 membrane thickness cancels out so that the thickness can be 229 neglected. Upon cancelation, it is noted the bending energy due to 230 the ADE contribution has a similar form to that of the local bend- 231 ing resistance and these energies could be lumped together [12]. 232 However, the ADE contribution is explicitly retained in the bend- 233 ing energy model in this work as a measurement to identify the 234 model shape.

Shearing Energy. The membrane shearing energy is the sum of 236 the potential energies of the springs comprising the spring net- 237 work. The springs employed are worm-like-chains (WLC) and, 238 therefore, the shearing energy has the following form:

$$
E_{S}=\frac{k_{B} T}{4 p} \sum_{j=1}^{N_{S}}\left[L_{c, j}\left(\frac{3 r_{j}^{2}-2 r_{j}^{3}}{1-r_{j}}-4 c_{1} r_{j}-c_{2}\right)\right]
$$

where $k_{B}$ is the Boltzmann constant, $T$ is the absolute temperature, 240 $p$ is the persistent length of the WLC elements, $j$ is spring index, 242 $N_{S}$ is the total number of springs, $L_{c, j}$ is the contour length of 243 WLC element $j$ and is defined as 2.3 times the reference element 244 length $L_{o, j}$, and $r_{j}$ is the ratio of the instantaneous element length 245 $L_{j}$ to the contour length of spring $j$, i.e., $r_{j}=L_{j} / L_{c, j}$, The WLC- 246 energy equation mentioned earlier differs from the conventional 247 form as two constants $c_{1}$ and $c_{2}$ are introduced so that the spring 248 elements have initial lengths. The constants $c_{1}$ and $c_{2}$ are set as 249 0.9673 and -0.9698 , respectively, from the definition of contour 250 length [30].

Initial and Reference Model Configurations. The RBC 252 model is used to predict the transformed shape of both vesicles 253

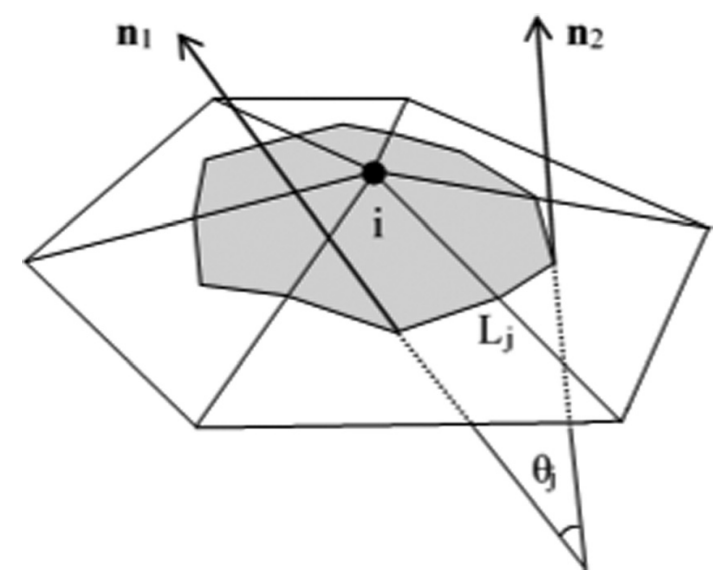

Fig. 2 Illustration of the curvature of particle $i$. The shaded area is the area occupied by particle $i$, i.e. $A_{i}, L_{j}$ is the length of edge $j, \mathrm{n}_{1}$ and $\mathrm{n}_{2}$ are the normal vectors to the two triangular elements which share edge $j$, and $\theta_{j}$ is the angle formed by these vectors, i.e., the included angle. 

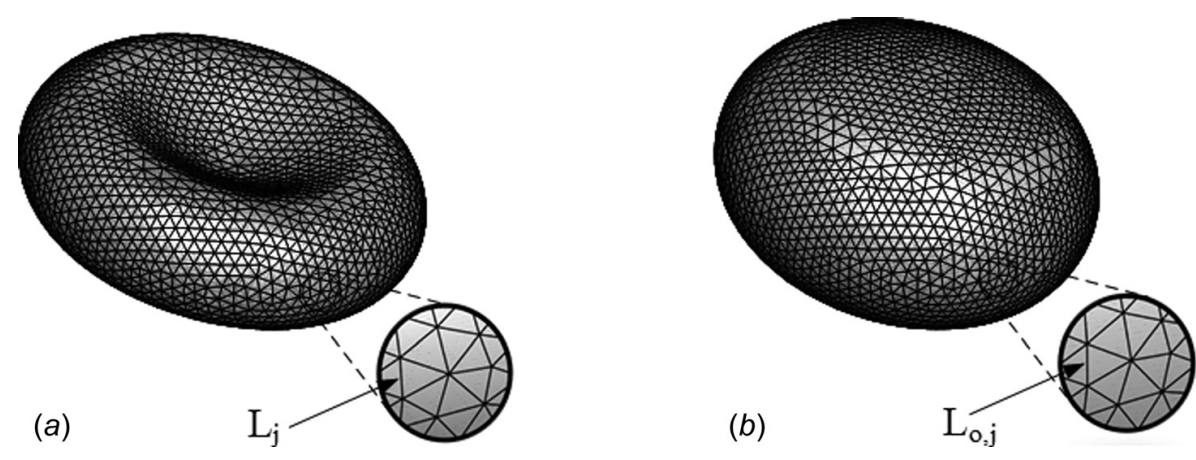

Fig. 3 Comparison of the geometry of (a) the initial discocyte with (b) the ellipsoid which is used to define the reference spring lengths, $L_{o, j}$, for the WLC springs. The magnified sections show the equivalent spring in both meshes.

254 and RBCs for a wide range of model volume and MAD. The ini255 tial vesicle/RBC configuration is always taken as a discocyte with 256 a volume, surface area, and MAD of approximately $94 \mu \mathrm{m}^{3}$, $257134 \mu \mathrm{m}^{2}$, and $0.332 \mu \mathrm{m}^{2}$, respectively. The reference configuration 258 is defined as follows: $V_{o}$ is the reference volume with a value 259 specified as the desired/target volume by the user, $A_{o}$ is the refer260 ence surface area and in this work is specified as the initial area of 261 the discocyte, $\Delta A_{\mathrm{o}}$ is the reference MAD with a value specified as 262 the desired/target MAD by the user, $C_{\mathrm{i}, o}$ is the reference nodal 263 curvature for node $i$ with all nodes assigned the same value 264 derived from $\Delta A_{o}$, i.e., Eq. (7), and $L_{o j}$ is the reference length for 265 spring $j$ on an ellipsoid [25] with a volume of $95 \%$ of a sphere 266 with the same surface area as the initial configuration, see Fig. 3 267 Experimental measurements suggest that the relaxed cytoskeleton 268 geometry is a quasi-sphere [31], while numerical predictions by 269 Lim et al. [25] suggest that the most likely relaxed geometry is an 270 ellipsoid [25], as described earlier and is thus used in this work.

RBC Shape Prediction. A transformed RBC shape is predicted using the principle of minimum free energy. In the RBC model, the free energy depends solely on the geometrical distribution of the particles since viscosity is neglected, i.e.

$$
E_{\text {Total }}=f\left(\mathbf{s}_{1}+\mathbf{s}_{2}+\ldots+\mathbf{s}_{i}\right)
$$

276 where $\mathbf{s}_{i}\left(x_{\mathrm{i}}, y_{\mathrm{i}}, z_{\mathrm{i}}\right)$ is the position vector of particle $i$. Therefore, the 277 internal force on a particle can be derived from the gradient of the 278 model free energy [32], i.e.

$$
\mathbf{F}_{i}=-\nabla E_{\text {Total }}\left(\mathbf{s}_{i}\right)
$$

$280 \quad$ or

$$
F_{i, x}=-\frac{\partial E_{\mathrm{Total}}}{\partial x_{i}}, \quad F_{i, y}=-\frac{\partial E_{\mathrm{Total}}}{\partial y_{i}}, \quad \text { and } \quad F_{i, z}=-\frac{\partial E_{\mathrm{Total}}}{\partial z_{i}}
$$

$$
E_{\text {Total }, n+1}=E_{\text {Total }, n}-\sum_{i=1}^{N_{P}}\left(\Phi_{i, n}\right)=E_{\text {Total }, n}-\sum_{i=1}^{N_{P}}\left(\mathbf{F}_{i, n} . \mathrm{d} \mathbf{s}_{i}\right)
$$

where $E_{\mathrm{Total}, n+1}$ and $E_{\mathrm{Total}, n}$ are the model free energies after and 292 before the incremental displacement of all the particles, respec- 293 tively. This equation implies that the model free energy reduces 294 on successive incremental displacements of the particles until the 295 particle forces reduce to zero. In this work, a transformed RBC 296 shape with a minimum free energy is predicted by iteratively 297 obtaining the internal force on each particle and incrementing 298 each particle position by $\mathrm{d} \mathbf{s}_{i}$ until for all nodes $\left|\mathbf{F}_{i}\right|<10^{-21} \mathrm{~N}$. The 299 incremental displacement $\mathrm{ds}_{i}$ is calculated as follows for each 300 node:

$$
\mathrm{ds}_{i}=\alpha \mathbf{F}_{i}
$$

where $\alpha\left(10^{6} \mathrm{~m} / \mathrm{N}\right)$ is a model constant with a magnitude equal to 303 $10 \%$ of the smallest spring-element length in the model.

\section{Results and Discussion}

This section contains results from two test cases. The first test 306 case involves the prediction of transformed vesicle shapes, while 307 the second one involves the prediction of transformed SDE RBC 308 shapes. In both test cases the transformed shapes are obtained for 309 a range of model volume and MAD.

Prediction of Vesicle Transformation. Physically, a vesicle is 311 equivalent to a RBC without the cytoskeleton. Therefore, in the 312 model, the free energy term related to the cytoskeleton, i.e., shear- 313 ing energy, is not required, with the vesicle transformation being 314 driven primarily by minimization of the bending energy. This is 315 an excellent test case to examine the accuracy and stability of the 316 bending model. In this subsection, the model is first validated by 317 comparing predicted vesicle shapes with experimental observa- 318 tions. Then, a vesicle transformation phase diagram is presented 319 showing transformed vesicle shapes for a range of model volume 320 and MAD, so that the capability of the model can be examined. 321

Vesicle Model Validation. Several predicted transformed vesi- 322 cle shapes, for various model volumes and MADs, are compared 323 with experimental observations [33] in Table 1. To make the 324 results transferable to vesicles with different surface areas, both 325 the volume and MAD are nondimensionalized with respect to a 326 sphere with the same surface area and membrane thickness as the 327 initial configuration. The nondimensionalized volume, known as 328 the reduced volume, is given by

$$
v=\frac{V_{\text {vesicle }}}{4 / 3 \pi R_{o}^{3}}
$$

where $V_{\text {vesicle }}$ is the vesicle volume and $R_{o}$ is the sphere radius 332 equal to $3.265 \mu \mathrm{m}$ for a surface area of $134 \mu \mathrm{m}^{2}$. The nondimen- 333 sional MAD is given by 
Table 1 Comparison of experimentally observed and numerically predicted transformed vesicle shapes using a fine and a coarse mesh. The experimental images are reprinted with permission from Refs. [34] and [35].

\begin{tabular}{|c|c|c|c|c|}
\hline \multicolumn{2}{|c|}{ Reduced } & \multirow[b]{2}{*}{ Experimentally observed } & \multirow[b]{2}{*}{ Numerically predicted (4636 elements) } & \multirow[b]{2}{*}{ Numerically predicted (1604 elements) } \\
\hline Vol. & MAD & & & \\
\hline 0.60 & 0.53 & & & \\
\hline 0.60 & 0.65 & & & \\
\hline 0.60 & 0.95 & & & \\
\hline 0.60 & 1.1 & & & \\
\hline 0.86 & 1.1 & & & \\
\hline 0.85 & 1.2 & & & \\
\hline 0.84 & 1.2 & & & \\
\hline
\end{tabular}

$$
\Delta a=\frac{\Delta A}{8 \pi R_{o} H}
$$

where $H$ is the membrane thickness with a value of $4 \mathrm{~nm}$. Note that the initial vesicle configuration, therefore, has $v \approx 0.645$ and $\Delta a \approx 1.01[34,35]$.

These predicted vesicle shapes were obtained using two different meshes: a fine mesh with 4636 triangular elements and a coarse mesh with 1604 elements. By observation, it can be seen that the predicted transformed shapes using either mesh are very similar to the observed vesicle shapes. Some differences are observable with the coarse-mesh models, however, and are due to the fact that the mesh elements are too large to correctly define the curved surface. The close agreement with the observed shapes indicates that the membrane bending energy is being correctly calculated and, importantly, the calculation is independent of 348 mesh density.

Phase Diagram of Vesicle Transformation. The fine-mesh 350 model described earlier was used to predict transformed vesicle 351 shapes for a range of reduced volume and MAD, see Fig. 4. It can 352 be seen from this figure that the predicted shapes fall within the 353 shape boundaries proposed by Seifert et al. [36]. These boundaries 354 separate the vesicle shapes into several categories, e.g., pear, 355 dumbbells, and stomatocytes. Seifert et al. investigated vesicle 356 shape transformation using a numerical model. In their study, the 357 phase diagram of vesicle transformation was developed using a 358 bilayer-coupling model, minimization of the bending energy only, 359 and with constraints similar to those employed in this work. Inter- 360 estingly, however, the membrane reference curvature was always 361 


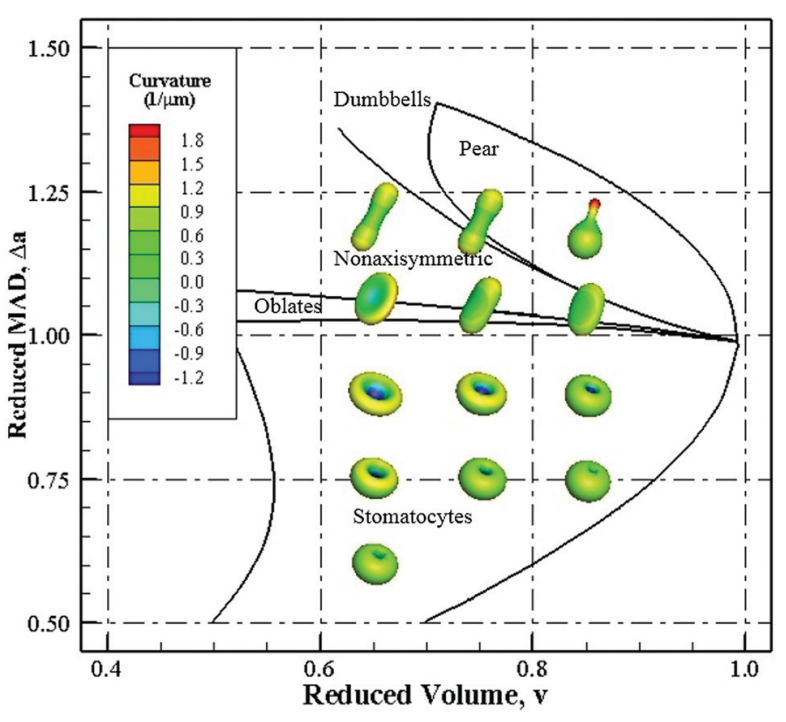

Fig. 4 The numerically predicted $\Delta a-v$ phase diagram of vesicle transformation. The predicted transformed shapes show contour maps of curvature. The boundaries shown are reproduced with permission from Seifert et al. [36]. 394 MAD. taken as zero. They showed using the phase diagram that vesicle shape transformation was continuous and that large and small MADs tended to induce outward and inward undulations, respectively.

In Fig. 4, it can be seen that, in general, oblates are the dominant transformed shapes for reduced volumes and reduced MADs similar to the initial configuration. Oblates are stable in a narrow range of reduced MAD; a small decrease leads to a shape shift toward stomatocytes, while a small increase leads to a shift toward stretched oblates which are similar to flattened dumbbells. Any further increase of the reduced MAD leads to a shape shift that depends on the vesicle reduced volume, i.e., a shift toward dumbbells for vesicles with small reduced volumes and a shift toward pears for vesicles with large reduced volumes.

In these simulations, the predicted vesicle shapes are mainly affected by the membrane bending energy model. The very good agreement with the observed shapes and the agreement with the previously defined boundaries indicate the bending model is appropriate for use in calculating the membrane bending energy. However, it should be noted that a bending calculation is limited by any severely deformed mesh elements. When vesicles are severely deformed, some triangular elements tend to collapse to severely stretched, needle like elements which can lead to the overlapping of neighboring triangular elements. As a result, the prediction of transformed vesicle shapes is restricted to a maximum reduced MAD of 1.3 [36].

Prediction of SDE Transformation. In this subsection, the RBC model is first validated by comparing predicted transformed RBC shapes both with experimental observations and with predicted results using a continuum RBC model. Then, an SDE transformation phase diagram is presented showing predicted ransformed shapes for a range of reduced volume and reduced

RBC Model Validation. Numerically predicted SDE transformed shapes are compared both with experimentally observed shapes and those predicted using a continuum RBC model [12,14] in Table 2. This comparison is for a reduced volume $v \approx 0.645$ and for a range of reduced MAD. By observation, it can be seen that a very good qualitative agreement is achieved. Overall, the predicted shapes accurately capture the detailed transformed geometries. In addition, it can be seen that, with shearing energy included, the model is capable of predicting the severely deformed 403 $\mathrm{RBCs}$ that occur for high reduced MADs. The good agreement 404 indicates that the SP-model can predict RBC mechanics upon 405 severe deformation and, importantly, the predicted results are 406 equivalent to those predicted using a continuum model 407 [12,37-39].

These transformed shapes were predicted using a mesh with 409 8610 triangular elements. This mesh density was chosen following 410 a mesh convergence study that had the aim of establishing the 411 mesh that would produce smooth and comparable shapes to those 412 observed and those predicted using the continuum model. Figure 413 5 gives some results from this study showing predicted echino- 414 cytes III with a reduced volume $v \approx 0.645$ and a reduced MAD 415 $\Delta a \approx 1.66$. These echinocytes were predicted using meshes with 416 2664, 4636, 8610, and 12,930 triangular elements. When using 417 meshes with less than 8610 elements some of the spicules are 418 very sharp tipped, see Figs. 5(a) and 5(b). However, when using 419 meshes with 8610 or more elements, all the spicules are very 420 smooth, and very little difference is found between the predicted 421 shapes. Also, it is interesting that these four echinocytes have a 422 comparable number of spicules; the one predicted with 4636 ele- 423 ments has 25 spicules while the others have 26 spicules.

Phase Diagram of SDE Transformation. The SP-RBC model 425 with a mesh of 8160 triangular elements was used to obtain the 426 phase diagram of SDE transformation. Figure 6 shows predicted 427 transformed RBC shapes with reduced MADs of one and above, 428 and at reduced volumes of $0.55,0.645,0.75$, and 0.85 . These pre- 429 dicted shapes correspond to the transformation of discocytes to 430 echinocytes I, II, and III. From observation, it can be seen that the 431 size of the echinocytes I spicules are slightly different for RBCs 432 with different reduced volumes. Low reduced-volume RBCs tend 433 to have higher number of periodic undulations on the rim, e.g., 11434 undulations for the RBC with a reduced volume of 0.55 , but eight 435 for the one with a reduced volume of 0.85 . It can also be seen that 436 the size of those undulations are approximately the same as the 437 thickness of the RBC rims. Therefore, high reduced-volume 438 RBCs, which have thick rims, have fewer undulations compared 439 with those with low reduced volumes. With further increases of 440 the MAD from echinocytes I, the undulations are differentiated 441 into small spicules distributed evenly over the entire RBC surface 442 to form echinocytes II. With an even further increase of the MAD, 443 echinocytes II transform to echinocytes III as more spicules are 444 formed and the oblate-shaped body of the echinocytes II is trans- 445 formed into an ellipsoidal shape. This body shape of the echino- 446 cyte III is affected by the choice of the reference spring length, 447 since the membrane shearing energy is the dominant energy deter- 448 mining the RBC shape transformation. In addition, it can be seen 449 in Fig. 6 that echinocytes II form in an extremely small range of 450 MAD in RBCs of high reduced volume. In those RBC models, the 451 echinocyte body is transformed into an ellipsoidal shape before 452 echinocytes II form and the spicules remain at the echinocyte rim. 453

In a similar fashion, Fig. 7 presents predicted transformed RBC 454 shapes with reduced MADs of one and below, and at reduced vol- 455 umes of $0.55,0.645,0.75$, and 0.85 . These predicted shapes corre- 456 spond to the shape transformations of discocytes to stomatocytes 457 I, II, and III. stomatocytes I form when one of the concave invagi- 458 nations vanishes, while the other becomes deeper. With further 459 decrease of the MAD, inward-directed undulations form in the 460 remaining concave invagination to form stomatocytes II, e.g., two 461 undulations form in the stomatocytes. Eventually, these undula- 462 tions merge to form a deep and elongated notch and form stomato- 463 cytes III.

It is very interesting to see that the transformed shapes in the 465 SDE transformation are very similar to those in the vesicle trans- 466 formation. First, oblates are the dominant shapes in a very narrow 467 range of the reduced MAD around $\Delta a=1.0$. Second, the shape 468 transformation is more severe for the model with high reduced 469 volume than the one with low reduced volume. Third, the shape 470 categories for both transformations are similar, e.g., stomatocyte 471 
Table 2 Comparison of experimentally observed and numerically predicted transformed RBC shapes in the SDE transformation. The predicted shapes are also compared with those predicted using a continuum model [12]. The experimental images are reprinted with permission from Refs. [37-39].

\begin{tabular}{|c|c|c|c|c|c|c|}
\hline \multirow{2}{*}{ Shape Category } & \multicolumn{2}{|c|}{ Reduced } & \multirow{2}{*}{$\begin{array}{c}\text { Experimentally } \\
\text { Observed }\end{array}$} & \multirow{2}{*}{$\begin{array}{c}\text { Numerically } \\
\text { Predicted } \\
\text { (SP-RBC) }\end{array}$} & \multirow{2}{*}{$\begin{array}{l}\text { Numerically } \\
\text { Predicted } \\
\text { (Continuum) }\end{array}$} & \\
\hline & Vol. & MAD & & & & \\
\hline Stomatocyte III & 0.645 & 0.76 & & & & \\
\hline Stomatocyte II & 0.645 & 0.83 & & & & $\begin{array}{c}(\mathbf{a} / \mu \mathbf{m}) \\
1.8\end{array}$ \\
\hline Stomatocyte I & 0.645 & 0.98 & & & & $\begin{array}{r}0.0 \\
-0.3\end{array}$ \\
\hline Discocyte & 0.645 & 1.02 & & & & \\
\hline Echinocyte I & 0.645 & 1.09 & & & & \\
\hline Echinocyte II & 0.645 & 1.47 & & & & \\
\hline Echinocyte III & 0.645 & 1.66 & & & & \\
\hline
\end{tabular}

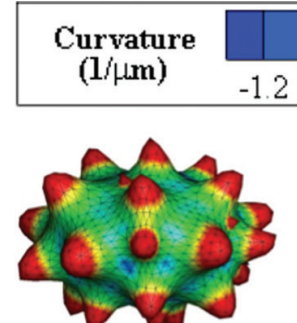

(a)

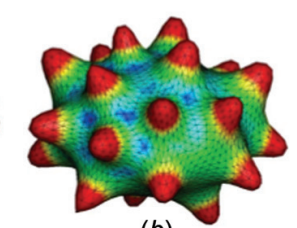

(b)

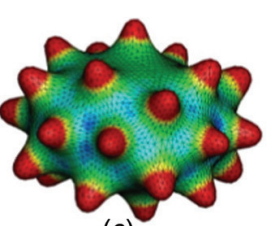

(c)

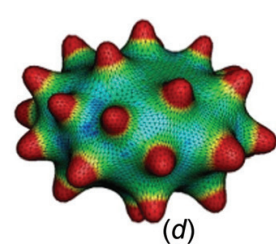

(d)

Fig. 5 Predicted echinocytes III with a reduced volume $v \approx 0.645$ and a reduced MAD $\Delta a \approx 1.66$. These transformed shapes were predicted using meshes with (a) 2664, (b) 4636, (c) 8610, and (d) 12,930 triangular elements. 


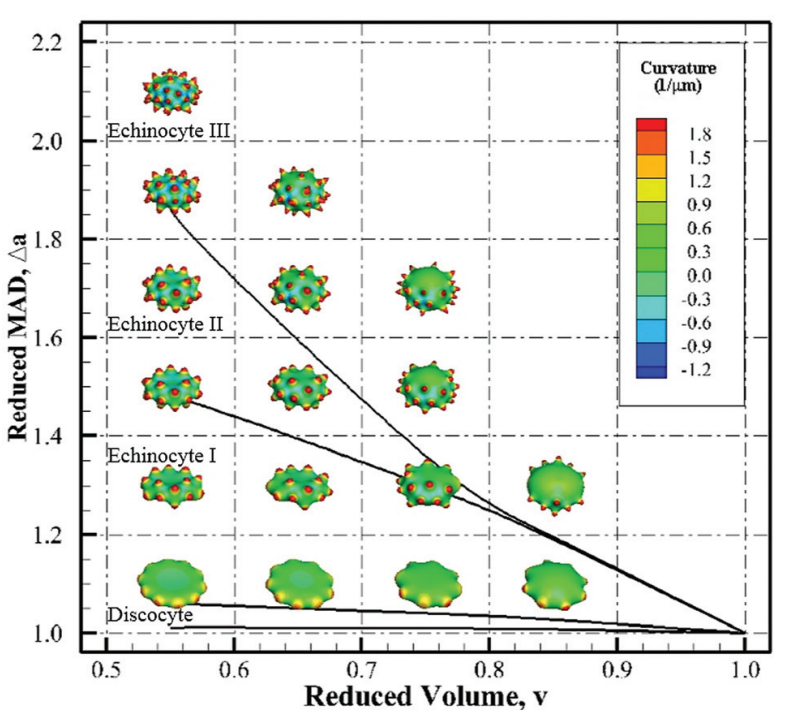

Fig. 6 The numerically predicted $\Delta a-v$ phase diagram of the SDE transformation from discocytes to echinocytes I, II, and III

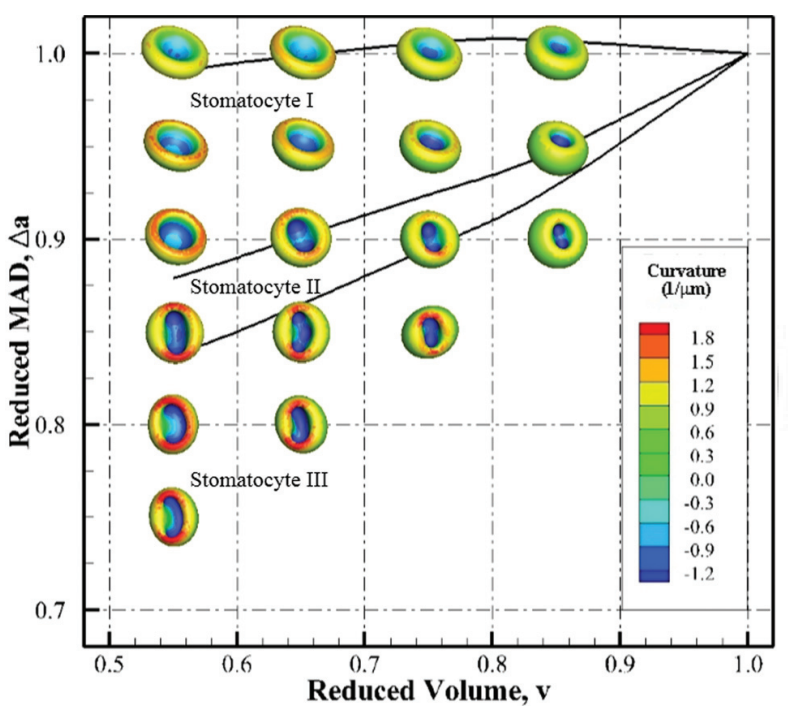

Fig. 7 The numerically predicted $\Delta a-v$ phase diagram of the SDE transformation from discocytes to stomatocytes I, II, and III vesicle and stomatocyte RBC, dumbbells and echinocyte II, and pears and echinocyte III. From observation, RBC shape boundaries can be defined in the SDE-transformation phase diagrams. These boundaries separate the predicted RBC geometries into shape categories, i.e., stomatocytes, discocytes, and echinocytes, see Figs. 6 and 7. However, it should be noted that the predicted RBC shapes may be metastable shapes due to the complexity of the RBC model [25] and that these boundaries are based on the assumptions and conditions employed in this work.

\section{Conclusion}

In this work, an enhanced SP-RBC model is proposed in order to extend the capability of conventional spring-network-based RBC models. In contrast with conventional SP-RBC models, in the enhanced model, the membrane bending energy is calculated using the surface curvature, thus rendering it independent of the mesh topology and the degree of model deformation. In addition, an ADE contribution to the bending energy is added for the first 488 time in a SP-RBC model.

The SP-RBC model is used to predict transformed RBC shapes. 490 While the results presented here can be considered as a follow-on 491 to the work of Lim et al. [25], there are several differences: in this 492 work, the RBC model employed is spring-network-based while 493 Lim's was continuum based, the accuracy of the membrane bend- 494 ing model is first established via a vesicle transformation study, 495 and the effect of model volume on the transformed shapes is 496 assessed, whereas in Lim et al., only one model RBC volume was 497 used. Critically, in this work, SDE RBC shape boundaries are pro- 498

posed for the first time which relate RBC shape categories to RBC 499 reduced volume and reduced MAD.

The results presented here show that the enhanced SP-RBC 501 model can accurately simulate vesicle and RBC SDE shape trans- 502 formations. Importantly, very good agreement is observed in com- 503 parisons of numerically predicted shapes with experimental 504 observations. In addition, the predicted SDE shapes are also very 505 similar to those predicted using a continuum RBC model indicat- 506 ing the equivalence of the present SP-RBC model with continuum 507 models.

\section{Acknowledgment}

509

The first author was supported by the Fiosraigh Research Schol- 510 arship provided by the Dublin Institute of Technology.

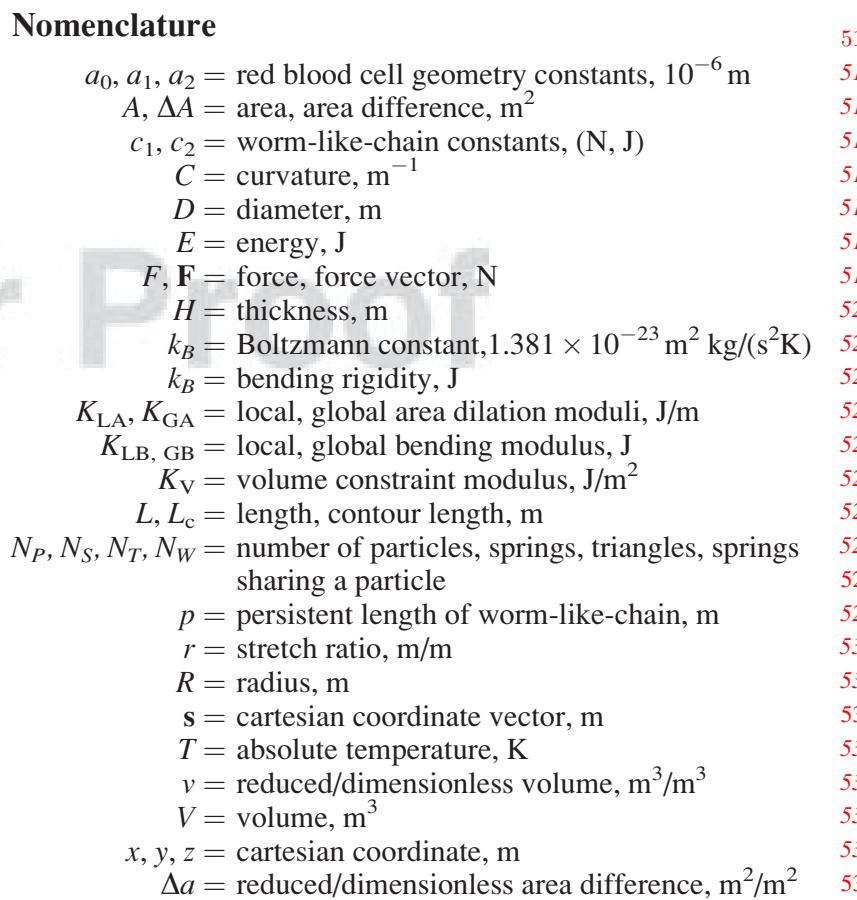

Greek Symbols

$$
\begin{aligned}
\Phi & =\text { force potential, } J \\
\theta & =\text { angle, radian } \\
\mu & =\text { micron, } 10^{-6} \\
\xi, \varsigma & =\text { normal vectors, } m
\end{aligned}
$$

Subscripts or Superscripts

$$
\begin{aligned}
\mathrm{AC} & =\text { area dilation constraint } \\
\mathrm{B} & =\text { bending constraint } \\
\mathrm{i} & =\text { particle index } \\
\mathrm{j} & =\text { spring index } \\
\mathrm{k} & =\text { triangle index } \\
\mathrm{o} & =\text { spontaneous/initial value }
\end{aligned}
$$$$
\mathrm{S}=\text { shearing constraint }
$$ 
Total $=$ total

$\mathrm{V}, \mathrm{VC}=$ volume, volume constraint

\section{Abbreviations widely used in text}

$\mathrm{ADE}=$ area difference elasticity

$\mathrm{MAD}=$ membrane area difference

$\mathrm{PM}=$ plasma membrane

$\mathrm{RBC}=$ red blood cell

$\mathrm{SDE}=$ stomatocyte-discocyte-echinocyte

$\mathrm{SK}=$ skalak

$\mathrm{SP}=$ spring particle

$\mathrm{WLC}=$ worm-like-chain

\section{Appendix}

According to the Helfrich bending model, the bending energy, $E_{B}$, contained in a thin membrane element of area $A$ at its neutral plane, see Fig. 8, is given by

$$
E_{B}=\frac{K_{B}}{2}\left(\mathrm{C}-C_{o}\right)^{2} A
$$

where $K_{B}$ is the bending modulus, $C$ is the curvature, $C_{o}$ is the curvature of a reference configuration, with both curvatures assumed constant over the surface. For the element shown in Fig. 8, the curvature is calculated as $C=1 / R_{1}+1 / R_{2}$, where $R_{1}$ and $R_{2}$ are the principal radii of curvature at the neutral plane.

For the membrane element, it can also be shown that the curvature can be calculated from the area difference between inner and outer surfaces of the membrane, $\Delta A$, i.e.,

$$
C=\frac{\frac{\Delta A}{H}}{A}
$$

where $H$ is the element thickness, assumed constant.

In our spring-particle model the membrane surface is discretized using a mesh of contiguous triangular elements, with the element boundaries considered the springs and the element nodes considered the particles. The model bending energy is calculated at the nodes/particles, and for each particle is the energy of a region of the membrane centered around that particle. Consider

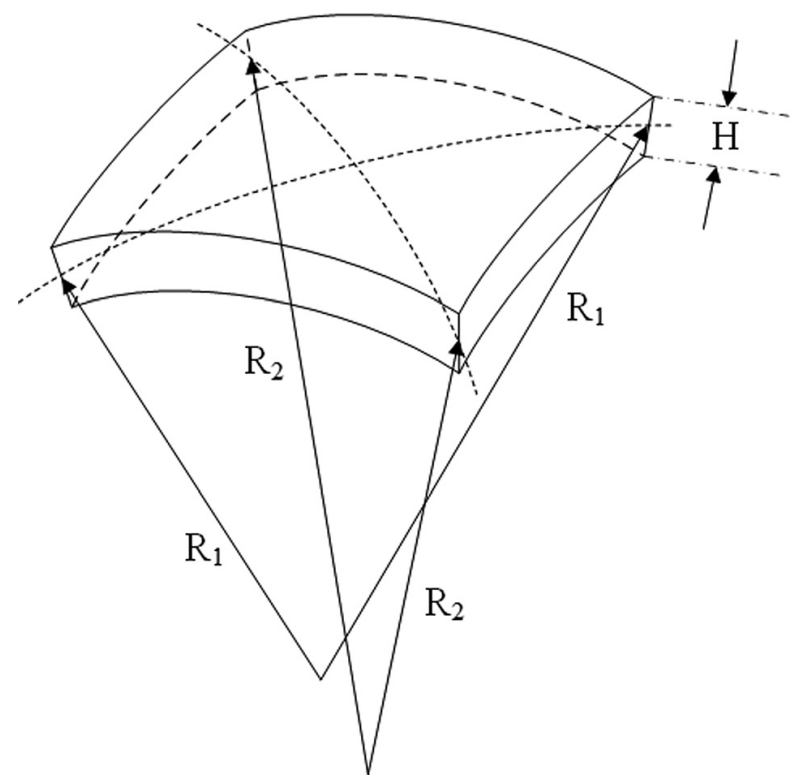

Fig. 8 A thin membrane element of area $A . R_{1}$ and $R_{2}$ are the principle radii of curvature at the neutral plane, and $H$ is the element thickness.

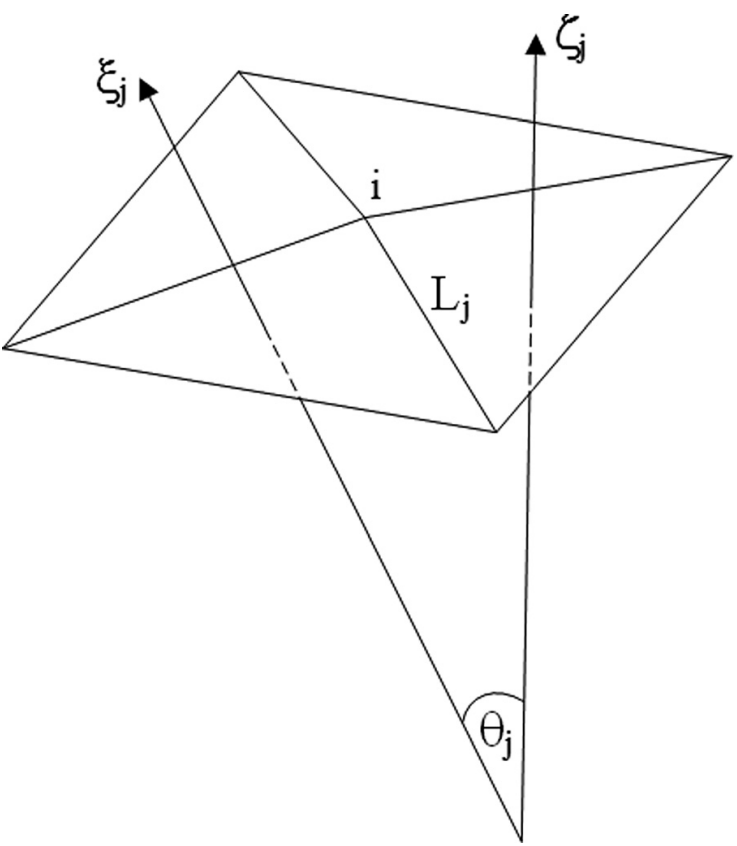

Fig. 9 Calculation of the model bending energy using curvature. $i$ is the particle index. For an edge $j \xi_{j}$ and $\zeta_{j}$ are the surface-normal vectors to the neighboring elements, $L_{j}$ is the edge length, and $\theta_{j}$ is the subtended angle.

the four triangular elements (of the overall surface mesh) shown 584 in Fig. 9 used to model the membrane element shown in Fig. 8, 585 with a combined area of $A$ and sharing the common particle $i .586$ These elements are assumed to be located at the neutral plane of 587 the membrane. Neighboring elements have a shared edge of length 588 $L_{j}$, where $j$ is the index of the edge. In addition, the surface-normal 589 vectors to neighboring elements form the included angle $\theta_{j}$. 590

Using Eq. (A1), the model bending energy for particle $i$ is cal- 591 culated as follows:

$$
E_{B, i}=\frac{K_{B}}{2}\left(C_{i}-C_{i, o}\right)^{2} A_{i}
$$

where $C_{\mathrm{i}}$ and $C_{\mathrm{i}, o}$ are the instantaneous and reference curvatures 593 at particle $i$, and $A_{\mathrm{i}}$ is the effective area of particle $i$ equal to one- 595 third of the total area of the elements which share the particle. 596 Using Eq. (A2), the curvature $C_{\mathrm{i}}$ is calculated as

$$
C_{i}=\frac{\frac{1}{2} \sum_{j=1}^{N_{w}}\left(\theta_{j} L_{j}\right)}{A_{i}}
$$

where $N_{W}$ is the total number of element edges meeting at particle 598 $i$. Equation (19) combined with Eq. (A4) are the equations 600 employed in this work to calculate the bending energy. Summing 601 the bending energies of all the particles gives the bending energy 602 of the entire membrane model.

For the case in Fig. 8, where one of the principal radii of curva- 604 ture, e.g., $R_{2}$, is infinitely large, the membrane element can be 605 modeled by two planar elements as shown in Fig. 10, with a 606 shared edge of length $L$ and a combined area $A$.

Using Eq. (A1), the model bending energy for these two ele- 608 ments is

$$
E_{B}=\frac{K_{B}}{2}\left(C-C_{o}\right)^{2} A
$$

with the model curvature given by

$$
C=\frac{\theta L}{A}
$$




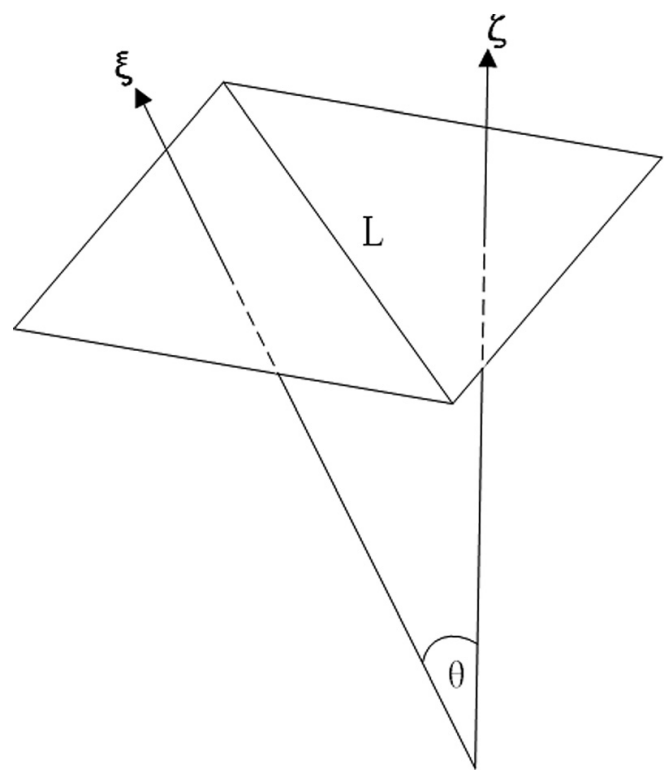

Fig. 10 Calculation of the model bending energy using the subtended angle. $\xi$ and $\zeta$ are the surface normal vectors, $L$ is the common-edge length, and $\theta$ is the subtended angle.

Therefore, the model bending energy can be calculated by substituting Eq. (A6) into Eq. (A5), i.e.,

$$
E_{B}=\frac{K_{B}}{2}\left(\frac{\left(\theta-\theta_{o}\right)^{2} L^{2}}{A}\right)
$$

616 where $\theta_{o}$ is the corresponding angle of the reference configuration.

617 The bending energy of the entire model is obtained by calculating 618 the bending energy of the element pairs.

619 Using Taylor's Theorem which states the following:

$$
\begin{aligned}
\cos \left(\theta-\theta_{o}\right)= & 1-\frac{1}{2 !}\left(\theta-\theta_{o}\right)^{2}+\frac{1}{4 !}\left(\theta-\theta_{o}\right)^{4} \\
& -\frac{1}{6 !}\left(\theta-\theta_{o}\right)^{6}+\cdots+\frac{(-1)^{n}}{(2 n) !}\left(\theta-\theta_{o}\right)^{2 n}
\end{aligned}
$$

620 the $\left(\theta-\theta_{o}\right)^{2}$ term in Eq. (A8) can be replaced so that the bending 622 energy can be written as

$$
\begin{aligned}
E_{B}= & K_{B}\left(\frac{L^{2}}{A}\right)\left[1-\cos \left(\theta-\theta_{o}\right)+\frac{1}{4 !}\left(\theta-\theta_{o}\right)^{4}\right. \\
& \left.-\frac{1}{6 !}\left(\theta-\theta_{o}\right)^{6}+\cdots+\frac{(-1)^{n}}{(2 n) !}\left(\theta-\theta_{o}\right)^{2 n}\right]
\end{aligned}
$$

628 If both of the model elements are equilateral triangles and if all 625 the element edge lengths remain constant during deformation, the 626 bending energy can be further simplified to

$$
\begin{aligned}
E_{B}= & K_{B}\left(\frac{L^{2}}{\frac{\sqrt{3}}{2} L^{2}}\right)\left[1-\cos \left(\theta-\theta_{o}\right)+\frac{1}{4 !}\left(\theta-\theta_{o}\right)^{4}\right. \\
& \left.-\frac{1}{6 !}\left(\theta-\theta_{o}\right)^{6}+\cdots+\frac{(-1)^{n}}{(2 n) !}\left(\theta-\theta_{o}\right)^{2 n}\right]
\end{aligned}
$$

628 or

$$
\begin{aligned}
E_{B}= & k_{B}\left[1-\cos \left(\theta-\theta_{o}\right)+\frac{1}{4 !}\left(\theta-\theta_{o}\right)^{4}\right. \\
& \left.-\frac{1}{6 !}\left(\theta-\theta_{o}\right)^{6}+\cdots+\frac{(-1)^{n}}{(2 n) !}\left(\theta-\theta_{o}\right)^{2 n}\right]
\end{aligned}
$$

630 where $k_{B}=2 K_{B} / \sqrt{3}$.
Equation (27), without the fourth and higher-order terms, is the 631 typical bending-energy model employed in conventional SP-RBC 632 models $[28,40]$, i.e.

$$
E_{B}=k_{B}\left[1-\cos \left(\theta-\theta_{o}\right)\right]
$$

From the derivation just above, it can be seen that this bending 633 model is an approximation to the Helfrich bending model [41] 636 with an error that is fourth order with respect to the angle differ- 637 ence between the instantaneous and reference configurations. 638 Importantly, this bending model was formulated based on two 639 assumptions. The first assumption is that one of the radii of curva- 640 ture of the membrane is infinitely large and the second is that the 641 model elements are nondeformable equilateral triangles. The first 642 assumption means the model is not capable of accurately predict- 643 ing the bending energy of surfaces with complex curvatures. In 644 addition, the second assumption means the model cannot accu- 645 rately predict the bending energy if the model undergoes deforma- 646 tion that leads to severely stretched triangular elements, as usually 647 happens with severely deformed models.

\section{References}

[1] Sherwood, L., 2010, Human Physiology: From Cells to Systems, Brooks/Cole, Cengage Learning, $\mathbf{\square}$, Australia.

[2] Skalak, R., Ozkaya, N., and Skalak, T. C., 1989, "Biofluid Mechanics," Annu. Rev. Fluid Mech., 21(1), pp. 167-204.

[3] Ruef, P., and Linderkamp, O., 1999, "Deformability and Geometry of Neonatal Erythrocytes With Irregular Shapes," Pediatr. Res., 45(1), pp. 114-119.

[4] Wolanskyj, A. P., 2013, "Benign Hematology," Mayo Clinic Internal Medicine Board Review, R. D. Ficalora, ed., Oxford University Press, Oxford, UK, pp. 652 497-511.

[5] Chen, Y., Cai, J., and Zhao, J., 2002, "Diseased Red Blood Cells Studied by Atomic Force Microscopy,” Int. J. Nanosci., 1(5\&6), pp. 683-688.

[6] Zehnder, L., Schulzki, T., Goede, J. S., Hayes, J., and Reinhart, W. H., 2008, "Erythrocyte Storage in Hypertonic (SAGM) or Isotonic (PAGGSM) 655 Conservation Medium: Influence on Cell Properties," Vox Sang., 95(4), pp. 656 280-287.

[7] Chabanel, A., Reinhart, W., and Chien, S., 1987, "Increase Resistance to Membrane Deformation of Shape-Transformed Human Red Blood Cells," Blood, 658 69(3), pp. 739-743.

[8] Waugh, R. E., 1996, "Elastic Energy of Curvature-Driven Bump Formation on Red Blood Cell Membrane," Biophys. J., 70(2), pp. 1027-1035.

[9] Gedde, M. M., Davis, D. K., and Huestis, W. H., 1997, "Cytoplasmic $p \mathrm{H}$ and Human Erythrocyte Shape,” Biophys. J., 72(3), pp. 1234-1246.

[10] Jan, K. M., and Chien, S., 1973, "Role of Surface Electric Charge in Red Blood Cell Interactions,” J. Gen. Physiol., 61(5), pp. 638-654.

[11] Hsu, R., Kanofsky, J. R., and Yachnin, S., 1980, "The Formation of Echinocytes by the Insertion of Oxygenated Sterol Compounds Into Red Cell Mem- 663 branes," Blood, 56(1), pp. 109-117.

[12] Lim, H. W. G., Wortis, M., and Mukhopadhyay, R., 2002, "Stomatocyte-Discocyte-Echinocyte Sequence of the Human Red Blood Cell: Evidence for the 665 Bilayer-Couple Hypothesis From Membrane Mechanics," Proc. Natl. Acad. 666 Sci. U. S. A., 99(26), pp. 16766-16769.

[13] Skalak, R., Tozeren, A., Zarda, R. P., and Chien, S., 1973, "Strain Energy Function of Red Blood Cell Membranes," Biophys. J., 13(3), pp. 245-264.

[14] Khairy, K., and Howard, J., 2011, "Minimum-Energy Vesicle and Cell Shapes Calculated Using Spherical Harmonics Parameterization," Soft Matter, 7(5), 669 pp. 2138-2143.

[15] Fedosov, D. A., Caswell, B., and Karniadakis, G. E., 2010, "A Multiscale Red Blood Cell Model With Accurate Mechanics, Rheology, and Dynamics," Bio- 67 phys. J., 98(10), pp. 2215-2225.

[16] Dupin, M. M., Halliday, I., Care, C. M., and Munn, L. L., 2008, "Lattice Boltzmann Modelling of Blood Cell Dynamics," Int. J. Comput. Fluid Dyn., 22(7), 673 pp. 481-492.

[17] Imai, Y., Nakaaki, K., Kondo, H., Ishikawa, T., Teck Lim, C., and Yamaguchi, T., 2011, "Margination of Red Blood Cells Infected by Plasmodium Falciparum 675 in a Microvessel," J. Biomech., 44(8), pp. 1553-1558.

[18] Dao, M., Li, J., and Suresh, S., 2006, "Molecularly Based Analysis of Deformation of Spectrin Network and Human Erythrocyte," Mater. Sci. Eng. C, 26(8), 677 pp. 1232-1244.

[19] Fedosov, D. A., Caswell, B., Suresh, S., and Karniadakis, G. E., 2011, "Quantifying the Biophysical Characteristics of Plasmodium-Falciparum- 679 Parasitized Red Blood Cells in Microcirculation" Proc Natl. Acad. Sci. U. S. 680

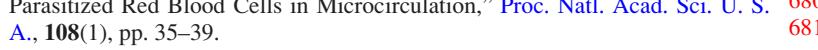

[20] Bow, H., Pivkin, I. V., Diez-Silva, M., Goldfless, S. J., Dao, M., Niles, J. C., Suresh, S., and Han, J., 2011, "A Microfabricated Deformability-Based 682 Flow Cytometer With Application to Malaria," Lab Chip, 11(6), pp. 683 $1065-1073$.

[21] Fedosov, D. A., Caswell, B., and Karniadakis, G., 2010, "Dissipative Particle Dynamics Modeling of Red Blood Cells," Computational Hydrodynamics of 685 Capsules and Biological Cells, C. Pozrikidis, ed., CRC Press, Boca Raton, FL, 686 pp. $183-218$. 
[22] Li, X., Vlahovska, P. M., and Karniadakis, G. E., 2013, "Continuum- and Particle-Based Modeling of Shapes and Dynamics of Red Blood Cells in Health and Disease," Soft Matter, 9(1), pp. 28-37.

[23] Pivkin, I., and Karniadakis, G., 2008, "Accurate Coarse-Grained Modeling of Red Blood Cells," Phys. Rev. Lett., 101(11), pp. 1-4.

[24] Alizadehrad, D., Imai, Y., Nakaaki, K., Ishikawa, T., and Yamaguchi, T., 2012, "Quantification of Red Blood Cell Deformation at High-Hematocrit Blood Flow in Microvessels," J. Biomech., 45(15), pp. 2684-2689.

[25] Lim, H. W. G., Wortis, M., and Mukhopadhyay, R, 2008, "Red Blood Cell Shapes and Shape Transformations: Newtonian Mechanics of a Composite Membrane," Soft Matter, G. Gompper, and M. Schick, eds., Wiley-VCH Verlag GmbH, Weinheim, Germany, pp. 83-203.

[26] Evans, E. A., and Fung, Y.-C., 1972, "Improved Measurements of the Erythrocyte Geometry,” Microvasc. Res., 4(4), pp. 335-347.

[27] Boey, S. K., Boal, D. H., and Discher, D. E., 1998, "Simulations of the Erythrocyte Cytoskeleton at Large Deformation-I: Microscopic Models.," Biophys. J., 75(3), pp. 1573-1583.

[28] Li, J., Dao, M., Lim, C. T., and Suresh, S., 2005, "Spectrin-Level Modeling of the Cytoskeleton and Optical Tweezers Stretching of the Erythrocyte.," Biophys. J. 88(5), pp. 3707-3719.

[29] Gompper, G., and Kroll, D. M., 1996, "Random Surface Discretizations and the Renormalization of the Bending Rigidity," J. Phys. I, 6(10), pp. 1305-1320.

[30] Chen, M., and Boyle, F. J., 2014, "Investigation of Membrane Mechanics Using Spring Networks: Application to Red-Blood-Cell Modelling," Mater. Sci. Eng. C, 43, pp. 506-516.

[31] Boal, D., 2012, "Introduction to the Cell," Mechanics of the Cell, Cambridge University Press, Cambridge, pp. 1-24.
[32] Housner, G. W., and Hudson, D. E., 1980, "Dynamics of a Particle," Applied Mechanics Dynamics, California Institute of Technology, Pasadena, CA, pp. 26-47. 705

[33] Svetina, S., and Zeks, B., 2002, "Shape Behavior of Lipid Vesicles as the Basis of Some Cellular Processes," Anat. Rec., 268(3), pp. 215-225.

[34] Käs, J., and Sackmann, E., 1991, "Shape Transitions and Shape Stability of Giant Phospholipid Vesicles in Pure Water Induced by Area-to-Volume 707 Changes," Biophys. J., 60(4), pp. 825-844.

[35] Käs, J., Sackmann, E., Podgornik, R., Svetina, S., and Žekš, B., 1993, "Thermally Induced Budding of Phospholipid Vesicles-A Discontinuous 709 Process," J. Phys. II, 3(5), pp. 631-645.

[36] Seifert, U., Berndl, K., and Lipowsky, R., 1991, "Shape Transformations of Vesicles: Phase Diagram for Spontaneous-Curvature and Bilayer-Coupling 71 Models," Phys. Rev. A, 44(2), pp. 1182-1202.

[37] Bessis, M. 1973, "Red Cell Shapes: An Illustrated Classification and Its

Rationale," Red Cell Shape, M. Bessis, R. I. Weed, and P. Leblond, eds., 713 Springer, Berlin, pp. 1-26.

[38] Brailsford, J. D., Korpman, R. A., and Bull, B. S., 1980, "Crenation and Cupping of the Red Cell: A New Theoretical Approach-Part II: Cupping," J. 71 Theor. Biol., 86(3), pp. 531-546.

[39] Jay, A. W., 1975, "Geometry of the Human Erythrocyte-I: Effect of Albumin on Cell Geometry," Biophys. J., 15(3), pp. 205-222.

[40] Pan, W., Fedosov, D. A., Caswell, B., and Karniadakis, G. E., 2011, "Predicting Dynamics and Rheology of Blood Flow: A Comparative Study of Multiscale 718 and Low-Dimensional Models of Red Blood Cells.," Microvasc. Res., 82(2), 719 pp. $163-170$.

[41] Helfrich, W., 1973, "Elastic Properties of Lipid Bilayers: Theory and Possible Experiments," Z. Naturforsch. C, 28(11-12), pp. 693-703. 15 711 ,

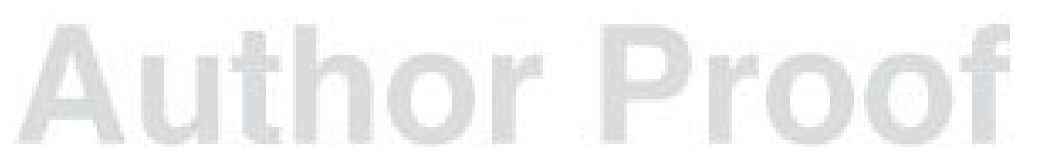

